\section{Glück im Englischen}

Die drei in allen regionalen Varietäten der Weltsprache Englisch am häufigsten verwendeten Substantive zur Bezeichnung von `Glück`als Glückserleben und Lebensglück sind happiness, (good) luck und (good) fortune sowie die abgeleiteten Adjektive happy, lucky und fortunate. Synonymwörterbücher listen unter happiness eine Reihe von Wohlbefindensgefühlen wie z.B. pleasure (`Vergnügen<, >Freude`), delight (`Entzücken`), felicity (gehoben für 'Glückseligkeit`), joy/fulness (`Freude`) oder cheerfulness (`Fröhlichkeit $`$ ) sowie blessedness, beatitude (beide meinen stilistisch erhöhte Glückseligkeit mit religiöser Konnotation; letzterer Begriff, wie auch bliss, mit shimmlisch assoziiert) und bliss (১Seligkeit $\iota$, 'Wonne ), deren jeweilige Aspekte von happiness auf zentrale Momente der dominanten Glücksauffassungen verweisen. So fällt bei blessedness und beatitude vor allem die bis ins 19. Jahrhundert wichtige und heute noch gelegentlich anzutreffende religiös-moralphilosophische Komponente ins Auge. Dagegen betonen die neueren Wörterbücher für happiness die emotionalen Nuancen des individuellen Glückserlebens.

Das Standardwörterbuch Oxford English Dictionary $(O E D)$ erläutert "happiness" (1) als "glückliche Fügung im Leben" und (2) als einen "angenehmen seelisch-geistigen Zustand «, der aus Erfolg oder dem Erreichen dessen, was als gut empfunden wird, resultiert. Aus dem heutigen Sprachgebrauch hat die Linguistin Anna Wierzbicka ein "Szenario" von "happiness « destilliert, das die individuelle Komponente des Begriffs (vgl. »the pursuit of happiness « in der amerikanischen Unabhängigkeitserklärung) herausstellt (Wierzbicka 1999, 51 ff.). Gegenüber dem Substantiv ist das oft in Redewendungen verwendete Adjektiv happy semantisch schwächer (54). Es drückt meist kein tiefes Glücksgefühl aus, sondern lediglich die $\mathrm{Zu}$ friedenheit mit etwas Gutem, wie in being happy with im Sinne von "zufrieden oder einverstanden sein" (52f.). Wierzbicka sieht diesen Prozess der Bedeutungserweiterung und damit verbundenen Intensitätsabschwächung als Teil einer generellen Dämpfung der Gefühle in der modernen englischsprachigen Gefühlskultur. Diese wird (besonders in den USA) von massenhaft verbreiteten Gefühlsanleitungen zu positive thinking, cheerfulness und fun dominiert (54), kann aber in ihrer Genese bereits im 19. Jahrhundert beobachtet werden (Gohrisch 2005, 120 ff.).
Etymologisch leiten sich happiness und happy von hap her, das als happ im Altnordischen für `Zufall steht, von wo es über das Alt- und Mittelenglische ins moderne Englisch gelangte, wo es (allerdings selten gebraucht) noch immer `Zufall und ‘zufälliges Ereignis bedeutet. Hap lebt auch im Verb to happen fort, das 'geschehen, sich (zufällig) ereignen` bedeutet.

Während in der sprachgeschichtlichen Entwicklung von happiness das Moment des Zufalls durch die Betonung des menschlichen Wollens und Fühlens zurückgedrängt wurde, dominiert es die Bedeutung von luck, für das u.a. fortune, chance, destiny, fate (meist negativ gebraucht) und (nur im amerikanischen Englisch) happenchance als Synonyme stehen. Luck bedeutet 'Schicksal, Geschick, Zufall und kann (wie fortune) sowohl als good luck (〉Glück`) oder bad luck (`Unglück, Pech`), d.h. als glückliche oder unglückliche Fügung des Schicksals, auftreten. Lucky und fortunate haben jedoch nur die positive Bedeutung >glücklich`. Etymologisch entstand luck durch Lautschwund aus dem Niederdeutschen geluk zu luk (vgl. das deutsche `Glück`). Zusätzlich zu luck kennt das Englische mit good/bad fortune noch ein weiteres, (über das Französische) aus dem Lateinischen entlehntes Wort für >un/glücklicher Zufall und >un/günstiger Umstand ‘ als einzelnes Geschehen. Des Weiteren führt das OED unter fortune die von Erfolg, d.h. von Wohlstand und Besitz definierte Stellung des Einzelnen auf, die sich in Formulierungen wie to make one's fortune (>sein Glück, d.h. ein Vermögen machen`) niederschlägt. Kulturgeschichtlich mag man hier das puritanisch-kalvinistische Erbe des anglo-amerikanischen Bürgertums durchscheinen sehen, das verstärkt seit dem 17. Jahrhundert beiderseits des Atlantiks Reichtum als Zeichen von Glück und damit der Gnadenwahl Gottes interpretierte.

\section{Literatur}

Gohrisch, Jana: Bürgerliche Gefühlsdispositionen in der englischen Prosa des 19. Jahrhunderts. Heidelberg 2005.

Oxford English Dictionary Online [OED]. Oxford 2009.

Wierzbicka, Anna: Emotion across Languages and Cultures. Diversity and Universals. Cambridge 1999.

Jana Gohrisch 